\title{
CONDUCTORS OF WILD EXTENSIONS OF LOCAL FIELDS, ESPECIALLY IN MIXED CHARACTERISTIC $(0,2)$
}

\author{
ANDREW OBUS
}

(Communicated by Matthew A. Papanikolas)

\begin{abstract}
If $K_{0}$ is the fraction field of the Witt vectors over an algebraically closed field $k$ of characteristic $p$, we calculate upper bounds on the conductor of higher ramification for (the Galois closure of) extensions of the form $K_{0}\left(\zeta_{p^{c}}, \sqrt[p]{a}\right) / K_{0}$, where $a \in K_{0}\left(\zeta_{p^{c}}\right)$. Here $\zeta_{p^{c}}$ is a primitive $p^{c}$ th root of unity. In certain cases, including when $a \in K_{0}$ and $p=2$, we calculate the conductor exactly. These calculations can be used to determine the discriminants of various extensions of $\mathbb{Q}$ obtained by adjoining roots of unity and radicals.
\end{abstract}

The purpose of this paper is to study the higher ramification filtrations of certain wild extensions of discrete valuation fields. Let $k$ be an algebraically closed field of characteristic $p>0$. We set $K_{0}=\operatorname{Frac}(W(k))$ and $K_{c}=K_{0}\left(\zeta_{p^{c}}\right)$, where $\zeta_{n}$ means a primitive $n$th root of unity. The main result is the determination of the higher ramification groups for Galois extensions of the form $K_{c}\left(\sqrt[2^{c}]{a}\right) / K_{0}$, where $c \geq 1$, $p=2$, and $a \in K_{0}$ (Theorem 5.1). In fact, we do not explicitly calculate all of the higher ramification groups, but rather the conductor of the extension, which is the highest index for which there exists a nontrivial higher ramification group for the upper numbering. Since the subextensions of extensions of this type have a form resembling that of the original extension, one can calculate the conductors of all the subextensions as well. One can then use properties of the higher ramification groups to show that this is enough to calculate all of the higher ramification groups of $K_{c}\left(\sqrt[2^{c}]{a}\right) / K_{0}$ (Proposition 1.3 and the introduction to $\$ 5$ ), which is in turn enough to calculate the different and discriminant ( Ser79, IV, Proposition 4, and VI, §3, Corollary 2]).

Additionally, we calculate an upper bound on the conductor of (the Galois closure of) any extension of the form $K=K_{c}(\sqrt[p]{a}) / K_{0}$, where $p$ is arbitrary and $a \in K_{c}$, but not necessarily in $K_{0}$ (Corollary 4.3). In certain situations, we get an exact value for the conductor (Proposition 4.2). Our calculations in this more general situation are in fact used in the proof of Theorem 5.1 (in particular, part (ii)(g)). Our techniques are reminiscent of those used by Viviani in [Viv04, where the assumptions are made that $a \in K_{0}$ and $p$ is odd. The main idea is to focus

Received by the editors October 2, 2011 and, in revised form, May 29, 2012.

2010 Mathematics Subject Classification. Primary 11S15, 11S20; Secondary 11R18, 11R20, $12 \mathrm{~F} 05,12 \mathrm{~F} 10$.

Key words and phrases. Higher ramification groups, local fields, conductor, cyclotomic extensions.

The author was supported by an NSF Postdoctoral Research Fellowship in the Mathematical Sciences. Final preparation of this paper took place at the Max-Planck-Institut für Mathematik in Bonn. 
on what we call p-primitive elements of a mixed characteristic discrete valuation field (Definition [3.2). Extensions obtained by taking roots of such elements are particularly amenable to having their higher ramification groups determined. We then proceed by writing $K$ as the compositum of extensions coming from roots of $p$-primitive elements and roots of unity and by using theorems about how higher ramification groups behave under taking the compositum (Lemmas 1.1, 1.2).

We have two main motivations. The first comes from Viv04. In it, Viviani calculates the higher ramification groups away from 2 of all Galois extensions $\mathbb{Q}\left(\zeta_{m}, \sqrt[m]{a}\right) / \mathbb{Q}$, so long as $m$ is odd and $a \in \mathbb{Q}$ satisfies a technical condition. He is able to reduce this to the study of the extensions $\mathbb{Q}_{p}\left(\zeta_{p^{c}}, \sqrt[p]{a}\right) / \mathbb{Q}_{p}$, where $p$ is odd and the $p$-valuation of $a \in \mathbb{Q}_{p}$ is either prime to $p$ or divisible by $p^{c}$ (hereafter, the "valuation condition"). Of course, one can make a base change to the maximal unramified extension $\mathbb{Q}_{p}^{u r}$ of $\mathbb{Q}_{p}$ without changing the higher ramification groups. Furthermore, since we are studying algebraic extensions, there is no harm in mak-

ing a further base change to the completion $C$ of $\mathbb{Q}_{p}^{u r}$. We note that if $k=\overline{\mathbb{F}_{p}}$, then $K_{0}=C$. Thus, the calculation of the higher ramification groups in Viv04 is equivalent to calculating the higher ramification groups of $K_{c}\left(\sqrt[p^{c}]{a}\right) / K_{0}$ when $p$ is odd, $k=\overline{\mathbb{F}_{p}}$, and $a \in K_{0}$ satisfies the valuation condition. Naturally, one would like a similar result when $p=2$, which is what Theorem 5.1 provides. Furthermore, we need no valuation condition on $a$ when $p=2$, although we are unfortunately not able to eliminate the valuation condition when $p$ is odd.

The second motivation comes from Obu09 and Obu10. Let $f: Y \rightarrow \mathbb{P}^{1}$ be a $G$-Galois cover of $\mathbb{P}^{1}$ branched at 0,1 , and $\infty$, a priori defined over the algebraic closure of $K_{0}$. If a $p$-Sylow subgroup of $G$ is of order $p$, then it turns out that $f$ can in fact be defined over a tame extension of $K_{0}$ (Wew03b). However, if a $p$-Sylow subgroup of $G$ is cyclic of order $p^{r}$, then the best that can be proven at the moment, especially when $p$ is small, is that $f$ can often be defined over a field of the form $K_{c}\left(\sqrt[p^{c}]{a}\right) / K_{0}$, where $a \in K_{c}$. In fact, even the stable model of $f$ can often be defined over such an extension. The bounds that we calculate on the conductors of these extensions (in particular, Corollary 4.4) are sufficient to yield aesthetically pleasing statements of the form "smaller cyclic $p$-Sylow subgroups lead to smaller conductors of the minimal field of definition over $K_{0}$ " (see Obu09, Theorem 1.3] and [Obu10, Theorem 1.1] for the specific statements).

After some basic results on how higher ramification groups act under compositums and towers of field extensions ( $\$ 1$ and $\$ 2$ ), we study the ramification behavior of prime order Kummer extensions and introduce the concept of $p$-primitive elements (\$3). The technical heart of the paper is $\$$, where we study the conductor of an extension $K_{c}\left(\sqrt[p^{c}]{a}\right) / K_{0}$ for $a \in K_{0}$ by breaking this extension up into extensions involving only roots of unity (well understood by [Ser79]) and prime order Kummer extensions. We put everything together in $\$ 5$ to prove Theorem 5.1

Conventions. Throughout this paper, the valuation $v_{K}$ on any discrete valuation field $K$ is normalized so that the valuation of a uniformizer is 1 .

\section{HighER RAMIFICATION FILTRATIONS}

We state here some facts from [Ser79, IV]. Let $K$ be a complete discrete valuation field with residue field $k$. If $L / K$ is a finite Galois extension of fields with Galois group $G$, then the group $G$ has a filtration $G=G_{0} \geq G_{i}\left(i \in \mathbb{R}_{\geq 0}\right)$ for the lower 
numbering. If $\pi_{L}$ is a uniformizer of $L$, this filtration is given by

$$
g \in G_{i} \Leftrightarrow v_{L}\left(g \pi_{L}-\pi_{L}\right) \geq i+1 .
$$

There is also a filtration $G=G^{0} \geq G^{i}(i \in \mathbb{R} \geq 0)$ for the upper numbering, defined by $G^{i}=G_{\psi_{L / K}(i)}$, where $\psi_{L / K}:[0, \infty) \rightarrow[0, \infty)$ is a certain monotonically increasing, piecewise linear function ([Ser79, IV, §3]). The inverse of $\psi_{L / K}$ is denoted $\phi_{L / K}$. Clearly $G^{\phi_{L / K}(j)}=G_{j}$ for $j \in[0, \infty)$. The subgroup $G_{i}$ (resp. $G^{i}$ ) is known as the ith higher ramification group for the lower numbering (resp. the upper numbering). If $H \leq G$, and $M=L^{H}$, then it follows from the definitions that the $i$ th higher ramification group $H_{i}$ for the lower numbering for $L / M$ is $G_{i} \cap H$. If, furthermore, $H$ is normal, then the $i$ th higher ramification group $(G / H)^{i}$ for the upper numbering for $M / K$ is $G^{i} /\left(G^{i} \cap H\right) \leq G / H$ ([Ser79, IV, Proposition 14]). We say that the lower numbering is invariant under subgroups, whereas the upper numbering is invariant under quotients.

The conductor of $L / K$, written $h_{L / K}$, is defined by

$$
h_{L / K}=\sup _{i \in[0, \infty)}\left(G^{i} \neq\{i d\}\right)
$$

(note that this differs by 1 from the definition of [Ser79, p. 228]). The highest lower jump of $L / K$, denoted $\ell_{L / K}$, is defined by

$$
\ell_{L / K}=\sup _{i \in[0, \infty)}\left(G_{i} \neq\{i d\}\right) .
$$

Of course, $\psi_{L / K}\left(h_{L / K}\right)=\ell_{L / K}$ and $\phi_{L / K}\left(\ell_{L / K}\right)=h_{L / K}$.

The following lemma is easy (for a proof, see e.g. Obu09, Lemma 2.3]).

Lemma 1.1. Given $L / K$ as above, let $M_{1}, \ldots, M_{\ell}$ be subfields of $L$ containing $K$ whose compositum is $L$. Then $h_{L / K}=\max _{i}\left(h_{M_{i} / K}\right)$.

Lemma 1.2. Given $L / K$ as above, let $M_{1}, M_{2}$, and $M_{3}$ be subfields of $L$ containing $K$, the compositum of any two of which is $L$. If $h_{M_{1} / K}>h_{M_{2} / K}$, then $h_{M_{3} / K}=$ $h_{M_{1} / K}=h_{L / K}$.

Proof. By Lemma 1.1 applied to $M_{1}$ and $M_{2}$, we have $h_{L / K}=h_{M_{1} / K}$. Then the same lemma, applied to $M_{2}$ and $M_{3}$, implies that $h_{L / K}=h_{M_{3} / K}$.

Proposition 1.3. Given $L / K$ a G-Galois extension as above, the higher ramification filtration is completely determined by knowing the conductor of each Galois extension $M / K$, where $K \subseteq M \subseteq L$.

Proof. Clearly it is enough to determine $G^{i}$ for all $i \geq 0$. For any normal subgroup $H \leq G$, invariance under quotients shows that $H \geq G^{i}$ iff $(G / H)^{i}=\{i d\}$, that is, if $h_{L^{H} / K}<i$ (the ramification filtration on $G / H$ corresponds to the extension $L^{H} / K$ ). If $H_{1}$ and $H_{2}$ are two normal subgroups of $G$, then $L^{H_{1} \cap H_{2}}$ is the compositum of $L^{H_{1}}$ and $L^{H_{2}}$. Thus Lemma 1.1 shows that there is a unique minimal normal subgroup $H \leq G$ such that $h_{L^{H} / K}<i$. Since $G^{i}$ is normal in $G$ ([Ser79, IV, Proposition 1]), we conclude that $G^{i}$ is, in fact, this minimal normal subgroup $H$.

The following result of Serre is proved only in the case $K_{0}=\mathbb{Q}_{p}$, but the proof immediately extends to the context below. 
Proposition 1.4 ([Ser79], IV, Proposition 18). Let $k$ be an algebraically closed field of characteristic $p$, let $K_{0}=\operatorname{Frac}(W(k))$, and for $n \geq 1$, let $K_{n}=K_{0}\left(\zeta_{p^{n}}\right)$. Identify $G:=\operatorname{Gal}\left(K_{n} / K_{0}\right)$ with $\left(\mathbb{Z} / p^{n}\right)^{\times}$. Then $G_{0}=G$, and for $0<i \leq p^{n-1}$, we have

$$
G_{i}=\left\{a \in G \mid a \equiv 1 \quad\left(\bmod p^{\left\lfloor\log _{p} i\right\rfloor+1}\right)\right\} .
$$

In particular, one calculates that $h_{K_{n} / K_{0}}=n-1$.

\section{RAMIFICATION FILTRATIONS IN TOWERS}

In this section, we give several results about how conductors act in towers.

Lemma 2.1. Let $K$ be a complete discrete valuation field with algebraically closed residue field. Let $K \subseteq L \subseteq M$ be finite Galois extensions such that $M$ is Galois over $K$. Then, either $h_{M / K}=h_{L / K}$, in which case $h_{M / L} \leq \ell_{L / K}$, or $h_{M / K}>h_{L / K}$, in which case

$$
\frac{1}{[L: K]}\left(h_{M / L}-\ell_{L / K}\right)=h_{M / K}-h_{L / K} .
$$

Proof. Let $G=\operatorname{Gal}(M / K), H=\operatorname{Gal}(M / L)$, and $G / H=\operatorname{Gal}(L / K)$. Since the upper numbering is invariant under taking quotients, $h_{M / K} \geq h_{L / K}$. Let $j$ be the greatest index such that $G_{j} \gtrless H_{j}$; i.e., $j$ is the greatest index such that $G_{j} \not \leq H$. Then $G_{i}=H_{i}$ for all $i>j$. Now, $G_{j}=G^{\phi_{M / K}(j)}$. By invariance under quotients, we have that

$$
\phi_{M / K}(j)=h_{L / K} .
$$

By applying $\psi_{L / K}$ to both sides of (2.1), and using the fact that $\phi_{M / K}=\phi_{L / K} \circ$ $\phi_{M / L}$ ([Ser79, IV, Proposition 15]), we obtain that

$$
\phi_{M / L}(j)=\ell_{L / K} .
$$

Suppose that $h_{M / K}=h_{L / K}$. Then, applying $\psi_{L / K}$, we get $\psi_{L / K}\left(h_{M / K}\right)=$ $\ell_{L / K}$. But

$$
\begin{aligned}
\psi_{L / K}\left(h_{M / K}\right) & =\left(\psi_{L / K} \circ \phi_{M / K}\right)\left(\ell_{M / K}\right)=\left(\psi_{L / K} \circ \phi_{L / K} \circ \phi_{M / L}\right)\left(\ell_{M / K}\right) \\
& =\phi_{M / L}\left(\ell_{M / K}\right) \geq \phi_{M / L}\left(\ell_{M / L}\right)=h_{M / L},
\end{aligned}
$$

so $h_{M / L} \leq \ell_{L / K}$.

Now suppose that $h_{M / K}>h_{L / K}$. Applying $\psi_{M / K}$ to both sides yields

$$
\ell_{M / K}>\psi_{M / K}\left(h_{L / K}\right)=j,
$$

the last equality following from (2.1). Since $G_{i}=H_{i}$ for $i>j$, we have that

$$
\ell_{M / L}=\ell_{M / K}
$$

It also follows that, for all $i>j$, we have $\left[G: G_{i}\right]=[L: K]\left[H: H_{i}\right]$; thus the slope of $\phi_{M / L}$ is $[L: K]$ times the slope of $\phi_{M / K}$ for arguments greater than $j$. So

$$
\frac{1}{[L: K]}\left(\phi_{M / L}\left(\ell_{M / K}\right)-\phi_{M / L}(j)\right)=\left(\phi_{M / K}\left(\ell_{M / K}\right)-\phi_{M / K}(j)\right) .
$$

By (2.3),$\phi_{M / L}\left(\ell_{M / K}\right)=\phi_{M / L}\left(\ell_{M / L}\right)=h_{M / L}$, and $\phi_{M / K}\left(\ell_{M / K}\right)=h_{M / K}$. Now substituting (2.1) and (2.2) into (2.4) gives the statement of the lemma. 
Corollary 2.2. Let $K$ be a complete discrete valuation field with algebraically closed residue field, and let $K \subset L \subseteq M$ be Galois field extensions so that $M$ is Galois over $K$. Assume that $[L: K]=p$. Then

$$
h_{M / K}=\max \left(h_{L / K}, \frac{p-1}{p} h_{L / K}+\frac{1}{p} h_{M / L}\right) .
$$

Proof. Since $h_{L / K}$ is equal to the (only) lower jump $\ell_{L / K}$ of $L / K$, Lemma 2.1 implies that either $h_{M / K}=h_{L / K}$ or $\frac{1}{p}\left(h_{M / L}-h_{L / K}\right)=h_{M / K}-h_{L / K}$. Solving for $h_{M / K}$ proves the corollary.

The next corollary generalizes [Ray99, Lemme 1.1.4].

Corollary 2.3. Let $K$ be a complete discrete valuation field with algebraically closed residue field, let $K^{\prime}$ be a $\mathbb{Z} / p$-extension of $K$, and let $L$ be any finite Galois extension of $K$. Write $L^{\prime}$ for the compositum of $L$ and $K^{\prime}$ in some algebraic closure of $K$ (we do not assume the extensions are linearly disjoint). Then, if $h_{L / K}>h_{K^{\prime} / K}$, we have $h_{L^{\prime} / K^{\prime}}=p h_{L / K}-(p-1) h_{K^{\prime} / K}$. If $h_{L / K} \leq h_{K^{\prime} / K}$, then $h_{L^{\prime} / K^{\prime}} \leq h_{K^{\prime} / K}$.

Proof. We draw a diagram of the situation as follows:

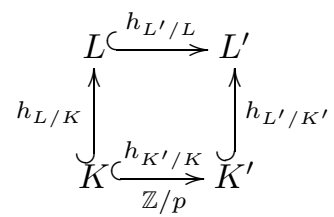

If $h_{L / K}>h_{K^{\prime} / K}$, then by Lemma 1.1. $h_{L^{\prime} / K}=h_{L / K}>h_{K^{\prime} / K}$. In this situation the corollary follows from Lemma 2.1 applied to the tower $K \subseteq K^{\prime} \subseteq L^{\prime}$, using the fact that $\ell_{K^{\prime} / K}=h_{K^{\prime} / K}$. If $h_{L / K} \leq h_{K^{\prime} / K}$, then, by Lemma 1.1] we have $h_{L^{\prime} / K}=h_{K^{\prime} / K}$. Then $h_{L^{\prime} / K^{\prime}} \leq \ell_{K^{\prime} / K}=h_{K^{\prime} / K}$ by Lemma 2.1 applied to the tower $K \subseteq K^{\prime} \subseteq L^{\prime}$.

Recall that $K_{0}$ is $\operatorname{Frac}(W(k))$, where $k$ is an algebraically closed field of characteristic $p$, and that $K_{r}=K_{0}\left(\zeta_{p^{r}}\right)$.

Corollary 2.4. Let $r \geq 1$, and let $M / K_{\ell}$ be a finite extension such that $M / K_{0}$ is Galois. Then

$$
h_{M / K_{0}}=\max \left(\ell-1, \ell-\frac{p}{p-1}+\frac{1}{(p-1) p^{\ell-1}}\left(h_{M / K_{\ell}}+1\right)\right) .
$$

Proof. By Proposition 1.4, the conductor of $K_{\ell} / K_{0}$ is $\ell-1$, and the greatest lower jump is $p^{\ell-1}-1$. So by Lemma 2.1] applied to the tower $K_{0} \subset K_{\ell} \subset M$, either $h_{M / K_{0}}=\ell-1$ or $\frac{1}{(p-1) p^{\ell-1}}\left(h_{M / K_{\ell}}-\left(p^{\ell-1}-1\right)\right)=h_{M / K_{0}}-(\ell-1)$. Solving for $h_{M / K_{0}}$ yields the corollary.

\section{PRIME order EXTENSIONS IN MIXED CHARACTERISTIC}

If $K$ is a mixed characteristic $(0, p)$ discrete valuation ring, then we write $e_{K}=$ $v_{K}(p)$, which is the absolute ramification index of $K$.

Lemma 3.1. Let $K$ be a finite extension of $K_{0}$, and suppose $a=1+t \in K$ with $v_{K}(t)<\frac{p}{p-1} e_{K}$. Then any pth root of a can be expressed (in an appropriate finite extension $L / K$ ) as $1+r$, where $v_{L}(r)=\frac{v_{L}(t)}{p}$. 
Proof. If $r \in L$ is as in the lemma, then $1+t=(1+r)^{p}=1+\sum_{i=1}^{p}\left(\begin{array}{c}p \\ i\end{array}\right) r^{i}$. Then $v_{L}(t) \geq \min _{1 \leq i \leq p}\left(v_{L}\left(\left(\begin{array}{c}p \\ i\end{array}\right) r^{i}\right)\right)$. Since $v_{L}(t)<\frac{p}{p-1} e_{L}$, we have $v_{L}(r)<\frac{1}{p-1} e_{L}$, which in turn implies that the minimum is realized for $i=p$. So $v_{L}(t)=v_{L}\left(r^{p}\right)$, from which the lemma follows.

Definition 3.2. Let $K / K_{1}$ be finite. If $a=1+t$ is an element of $K$ such that either $p \nmid v_{K}(a)$ or $0<v_{K}(t)<\frac{p}{p-1} e_{K}$ and $p \nmid v_{K}(t)$, then we will say that $a$ is p-primitive for $K$.

Lemma 3.3. Let $K$ be a finite extension of $K_{1}$, and let $L / K$ be a (nontrivial) $\mathbb{Z} / p$-extension.

(i) We have $L=K(\sqrt[p]{a})$, where a is p-primitive for $K$.

(ii) For $a$ and $t$ as in (i), the conductor $h_{L / K}$ is $\frac{p}{p-1} e_{K}-v_{K}(t)$.

Proof of (i). By Kummer theory, we know we can find $a$ such that $L \cong K(\sqrt[p]{a})$. In choosing $a$, we are free to multiply by elements of $\left(K^{\times}\right)^{p}$. Thus we can assume that $0 \leq v_{K}(a)<p$. If $v_{K}(a)>0$, then $a$ is $p$-primitive for $K$. If $v_{K}(a)=0$, we can use the fact that $k$ is algebraically closed to multiply $a$ by a $p$ th power so that it is congruent to 1 modulo a uniformizer. So write $a=1+t$, where $v_{K}(t)>0$. If $v_{K}(t) \geq \frac{p}{p-1} e_{K}$, then $a$ is a $p$ th power in $K($ Epp73, $\left.\$ 0.3]\right)$, contradicting the nontriviality of $L / K$. So $v_{K}(t)<\frac{p}{p-1} e_{K}$. If $p \nmid v_{K}(t)$, then $a$ is $p$-primitive for $K$. If $p \mid v_{K}(t)$, then write $t=\left(\pi_{K}\right)^{p \nu} w$, where $\pi_{K}$ is a uniformizer of $K$ and $v_{K}(w)=0$. Let $y \in K$ be such that $v_{K}\left(y^{p}+w\right)>0$ (we can find such a $y$ because $k$ is algebraically closed). If $a^{\prime}=a\left(1+\left(\pi_{K}\right)^{\nu} y\right)^{p}$, then $a^{\prime}=1+t^{\prime}$ with $v_{K}\left(t^{\prime}\right)>v_{K}(t)$. So replace $a$ by $a^{\prime}$ and $t$ by $t^{\prime}$ and repeat until $a$ is $p$-primitive for $K$. This process must terminate eventually, as the valuation of $t$ is bounded by $\frac{p}{p-1} e_{K}$.

Proof of (ii) (cf. [Viv04, Theorems 5.6 and 6.3]). We first calculate a uniformizer $\pi_{L}$ of $L$. Then, if $\sigma$ is a generator of $\operatorname{Gal}(L / K)$, we will determine $v\left(\sigma\left(\pi_{L}-\pi_{L}\right)\right)$. Let $\sqrt[p]{a}$ be a choice of $p$ th root such that $\sigma(\sqrt[p]{a})=\zeta_{p} \sqrt[p]{a}$.

Suppose $p \nmid v_{K}(a)$. Choose integers $m$ and $n$ such that $m p+n v_{K}(a)=1$. Thus $v_{L}\left(\pi_{K}^{m} a^{n / p}\right)=1$, so we set $\pi_{L}=\pi_{K}^{m} a^{n / p}$. Since $v_{L}\left(\zeta_{p}-1\right)=\frac{1}{p-1} e_{L}$, we have $v_{L}\left(\sigma\left(\pi_{L}\right)-\pi_{L}\right)=m p+v_{L}\left(a^{n / p}\right)+\frac{1}{p-1} e_{L}$. Since $L / K$ is a $\mathbb{Z} / p$-extension, the definition of the conductor gives $h_{L / K}=m p+\frac{n}{p} v_{L}(a)+\frac{1}{p-1} e_{L}-1=\frac{p}{p-1} e_{K}$. Since $v_{K}(t)=0$, this proves the proposition in this case.

Now, suppose $p \nmid v_{K}(t)>0$. By Lemma 3.1, $v_{L}(\sqrt[p]{a}-1)=\frac{v_{L}(t)}{p}=v_{K}(t)$. Since $p \nmid v_{K}(t)$, there exist $m, n \in \mathbb{Z}$ such that $m p+n v_{K}(t)=1$. We can even require that $0<n<p$. Then $\pi_{L}:=\pi_{K}^{m}(\sqrt[p]{a}-1)^{n}$ is a uniformizer of $L$.

Computing, we find

$$
\begin{aligned}
\sigma\left(\pi_{L}\right)-\pi_{L} & =\pi_{K}^{m}\left(\left(\sqrt[p]{a}-1+\left(\zeta_{p}-1\right) \sqrt[p]{a}\right)^{n}-(\sqrt[p]{a}-1)^{n}\right) \\
& =\pi_{K}^{m}\left(\sum_{i=0}^{n-1}\left(\begin{array}{c}
n \\
i
\end{array}\right)(\sqrt[p]{a}-1)^{i}\left(\left(\zeta_{p}-1\right) \sqrt[p]{a}\right)^{n-i}\right) .
\end{aligned}
$$

By assumption, $v_{L}\left(\zeta_{p}-1\right)=\frac{p}{p-1} e_{K}>v_{K}(t)=\frac{v_{L}(t)}{p}=v_{L}(\sqrt[p]{a}-1)$. Also, since $n<$ $p$, it follows that $\left(\begin{array}{c}n \\ i\end{array}\right)$ has valuation 0 . Thus all terms in the sum above have different valuations, and the term of lowest valuation corresponds to $i=n-1$. Applying $v_{L}$ 
to this term gives $m p+(n-1) v_{K}(t)+\frac{1}{p-1} e_{L}=1+\frac{p}{p-1} e_{K}-v_{K}(t)$. Since $L / K$ is a $\mathbb{Z} / p$-extension, the definition of the conductor gives $h_{L / K}=\frac{p}{p-1} e_{K}-v_{K}(t)$.

Lemma 3.4. If $L \cong K(\sqrt[p]{a})$ is a $\mathbb{Z} / p$-extension of $K$ with $a=1+t$, then $h_{L / K} \leq$ $\frac{p}{p-1} e_{K}-v_{K}(t)$, with equality holding iff a is p-primitive for $K$. In particular, if $a^{\prime}=1+t^{\prime}$ is p-primitive for $K$ and $a / a^{\prime}$ is a pth power in $K$, then $v_{K}\left(t^{\prime}\right) \geq v_{K}(t)$.

Proof. The proof of Lemma 3.3 shows that if $a=1+t$ is not $p$-primitive for $K$, then one can find $a^{\prime} p$-primitive for $K$ such that $a^{\prime}=1+t^{\prime}$ with $v_{K}\left(t^{\prime}\right)>v_{K}(t)$, and $\frac{a}{a^{\prime}}$ is a $p$ th power in $K$. The lemma then follows from Lemma 3.3 .

Corollary 3.5. Let $L_{0}$ be a finite extension of $K_{1}$. Let $a=1+t$ be p-primitive for $L_{0}$. For $i>0$, write $L_{i}=L_{0}(\sqrt[p]{a})$. Then $\left[L_{c}: L_{0}\right]=p^{c}$ for $c>0$, and for $0<i \leq c$, the conductor of $L_{i} / L_{i-1}$ is $\frac{p^{i}}{p-1} e_{L_{0}}-v_{L_{0}}(t)$.

Proof. If $p \nmid v(a)$, then clearly $\left[L_{c}: L_{0}\right]=p^{c}$ and $v_{L_{0}}(t)=0$. Also, $p \nmid v_{L_{i-1}}\left(\sqrt[p^{i-1}]{a}\right)$. Then Lemma 3.3 shows that $h_{L_{i} / L_{i-1}}=\frac{p}{p-1} e_{L_{i-1}}=\frac{p^{i}}{p-1} e_{L_{0}}$.

Suppose $v(a)=0$ and $p \nmid v(t)>0$. Since $L_{0}$ contains the $p$ th roots of unity, we know that $L_{1} / L_{0}$ is Galois. So we apply Lemma 3.3 to see that $\left[L_{1}: L_{0}\right]=p$ and $h_{L_{1} / L_{0}}$ is as desired. Choose a $p$ th root $\sqrt[p]{a}$. By Lemma 3.1, we have that $v_{L_{1}}(\sqrt[p]{a}-1)=\frac{v_{L_{1}}(t)}{p}=v_{L_{0}}(t)$, which is prime to $p$. So $\sqrt[p]{a}$ is $p$-primitive for $L_{1}$. Applying Lemma 3.3 to $L_{2} / L_{1}$ and $\sqrt[p]{a}$ shows that $\left[L_{2}: L_{1}\right]=p$ and

$$
h_{L_{2} / L_{1}}=\frac{p}{p-1} e_{L_{1}}-v_{L_{1}}(\sqrt[p]{a}-1)=\frac{p^{2}}{p-1} e_{L_{0}}-v_{L_{0}}(t),
$$

as desired. Repeating this process up to reaching $L_{c}$ yields the corollary. Note that in each case, $L_{i} / L_{i-1}$ is Galois.

\section{Conductors of a certain class of metabelian extensions}

Recall that $K_{r}=K_{0}\left(\zeta_{p^{r}}\right)$. Write $v_{r}$ for the normalized valuation on $K_{r}$ such that a uniformizer has valuation 1 , that is, $v_{r}=v_{K_{r}}$.

Lemma 4.1. Choose integers $\ell$ and $c$ such that $1 \leq \ell \leq c$. Let $a=1+t \in K_{\ell}$ such that $a$ is p-primitive for $K_{\ell}$. Then the conductor of $K_{c}(\sqrt[p c]{a})$ over $K_{\ell}(\sqrt[p]{a})$ is less than or equal to $p^{c+\ell-1}-v_{\ell}(t)$, which is the conductor of $K_{\ell}\left(\sqrt[p^{c}]{a}\right)$ over $K_{\ell}\left(\sqrt[p^{c-1}]{a}\right)$.

Proof. If $c=\ell$, the lemma follows from Corollary 3.5 so assume $c>\ell$. For each $i, 0 \leq i \leq c$, let $h_{i}$ be the conductor of $K_{c}(\sqrt[p i]{a})$ over $K_{\ell}(\sqrt[p]{a})$. Then, using Proposition 1.4. one calculates $h_{0}=p^{\ell-1}((c-\ell)(p-1)+1)-1$. Furthermore, let $a_{i}$ be the conductor of $K_{\ell}(\sqrt[p i]{a})$ over $K_{\ell}(\sqrt[p i-1]{a})$. By Corollary 3.5 we have $a_{i}=p^{i+\ell-1}-v_{\ell}(t)$. Note that $v_{\ell}(t)<\frac{p}{p-1} e_{K_{\ell}}=p^{\ell}$. We must show that $h_{c} \leq a_{c}$. Our diagram of field extensions and conductors looks like this:

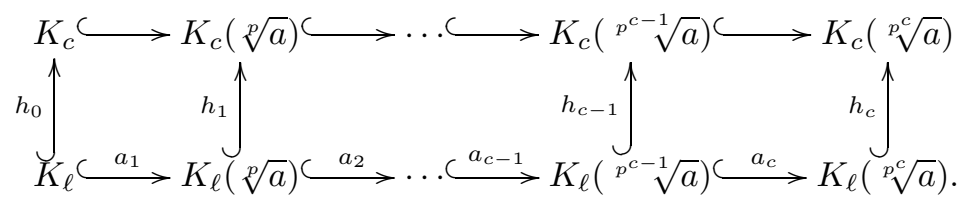


If there exists an $i, 0 \leq i<c$, such that $h_{i} \leq a_{i+1}$, then repeated application of Corollary 2.3 shows that $h_{c} \leq a_{c}$. So assume otherwise. Then we have the chain of (in)equalities below (the first comes from repeated application of Corollary 2.3):

$$
\begin{aligned}
h_{c} & =p^{c} h_{0}-p^{c-1}(p-1) a_{1}-p^{c-2}(p-1) a_{2}-\cdots-p(p-1) a_{c-1}-(p-1) a_{c} \\
& =p^{c} h_{0}-c(p-1)\left(p^{c+\ell-1}\right)+\left(p^{c}-1\right) v_{\ell}(t) \\
& =p^{c+\ell-1}(1-\ell(p-1))+\left(p^{c}-1\right) v_{\ell}(t)-p^{c} \\
& =a_{c}-\ell(p-1)\left(p^{c+\ell-1}\right)+p^{c} v_{\ell}(t)-p^{c} \\
& <a_{c}-\ell(p-1)\left(p^{c+\ell-1}\right)+p^{c+\ell}-p^{c} \\
& =a_{c}+p^{c+\ell}\left(1-\frac{\ell(p-1)}{p}-\frac{1}{p^{\ell}}\right) \\
& \leq a_{c} .
\end{aligned}
$$

Proposition 4.2. Choose integers $\ell \geq 1$ and $c \geq 1$. Let $a=1+t \in K_{\ell}$ such that a is p-primitive for $K_{\ell}$. Write $K=K_{\max (\ell, c)}\left(\sqrt[p^{c}]{a}\right)$. Then $K$ is Galois over $K_{\ell}$. Write $L$ for the Galois closure of $K$ over $K_{0}$.

(i) The conductor of $K / K_{\ell}$ is $(c(p-1)+1) p^{\ell-1}-v_{\ell}(t)$.

(ii) The conductor of $L / K_{0}$ is $\max \left(\ell-1, c+\ell-1-\frac{v_{\ell}(t)-1}{p^{\ell-1}(p-1)}\right)$.

Proof. The extension $K / K_{\ell}$ is clearly Galois. Let the $a_{i}$ be defined as in the proof of Lemma 4.1. Then as in that proof, we have $a_{i}=p^{i+\ell+1}-v_{\ell}(t)$.

Proof of (i). If $c \geq \ell$, then by Corollary 2.2 applied to $K_{\ell}(\sqrt[p-1]{a}) \subseteq K_{\ell}(\sqrt[p c]{a}) \subseteq K$, and using Lemma 4.1, we have that the conductor of $K$ over $K_{\ell}(\sqrt[p-1]{a})$ is $a_{c}$. If $c<\ell$, then the conductor of $K$ over $K_{\ell}(\sqrt[p-1]{a})$ is $a_{c}$ by definition. In both cases, applying Corollary 2.2 repeatedly to the extensions $K_{\ell}(\sqrt[p-1]{a}) \subseteq K_{\ell}(\sqrt[p]{a}) \subseteq K$ as $i$ ranges from $c-1$ to 1 , we obtain that the conductor of $K / K_{\ell}$ is

$$
\frac{1}{p^{c-1}} a_{c}+\sum_{i=1}^{c-1} \frac{p-1}{p^{i}} a_{i} .
$$

This is equal to $(c(p-1)+1) p^{\ell-1}-v_{\ell}(t)$.

Proof of (ii). Since $K$ is Galois over $K_{\ell}$, its Galois closure $L$ over $K_{0}$ is a compositum of conjugate extensions, each with the same conductor over $K_{\ell}$. By Lemma 1.1 and part (i) of this proposition, $h_{L / K_{\ell}}=h_{K / K_{\ell}}=(c(p-1)+1) p^{\ell-1}-v_{\ell}(t)$. By Corollary 2.4, we obtain

$$
h_{L / K_{0}}=\max \left(\ell-1, c+\ell-1-\frac{v_{\ell}(t)-1}{p^{\ell-1}(p-1)}\right) .
$$

Corollary 4.3. Choose integers $\ell \geq 1$ and $c \geq 1$. Let $\alpha \in K_{\ell}$, not necessarily p-primitive for $K_{\ell}$, with $v_{\ell}(\alpha) \geq 0$. Write $L$ for the Galois closure of $K:=K_{\max (\ell, c)}(\sqrt[p c]{\alpha})$ over $K_{0}$. We know $\alpha=\alpha^{\prime} \beta^{p}$, with either $\alpha^{\prime}=1$ or $\alpha^{\prime}$ p-primitive for $K_{\ell}$, and $\beta \in K_{\ell}$ with $v_{\ell}(\beta) \geq 0$. Write $\alpha^{\prime}=1+t_{\alpha^{\prime}}$ and $\beta=1+t_{\beta}$. 
Then

$$
\begin{aligned}
h_{L / K_{0}} & \leq \\
\mu & :=\max \left(\max (\ell, c)-1, c+\ell-1-\frac{v_{\ell}\left(t_{\alpha^{\prime}}\right)-1}{p^{\ell-1}(p-1)}, c+\ell-2-\frac{v_{\ell}\left(t_{\beta}\right)-1}{p^{\ell-1}(p-1)}\right) .
\end{aligned}
$$

Proof. It is clear that $L$ can be embedded into the Galois closure of $K_{\max (\ell, c)}\left(\sqrt[p^{c}]{\alpha^{\prime}}\right.$, $\sqrt[p^{c-1}]{\beta}$ ) over $K_{0}$. By Lemma 1.1, it suffices to show that the Galois closures $L^{\prime}$ of $K_{\max (\ell, c)}\left(\sqrt[p^{c}]{\alpha^{\prime}}\right)$ and $L^{\prime \prime}$ of $K_{\max (\ell, c-1)}\left(\sqrt[p^{c-1}]{\beta}\right)$ over $K_{0}$ satisfy $h_{L^{\prime} / K_{0}} \leq \mu$ and $h_{L^{\prime \prime} / K_{0}} \leq \mu$. By Proposition 4.2 (ii),

$$
h_{L^{\prime} / K_{0}}=\max \left(\ell-1, c+\ell-1-\frac{v_{\ell}\left(t_{\alpha^{\prime}}\right)-1}{p^{\ell-1}(p-1)}\right) \leq \mu
$$

when $\alpha^{\prime}$ is $p$-primitive for $K_{\ell}$. By Proposition 1.4, $h_{L^{\prime} / K_{0}}=\max (\ell, c)-1 \leq \mu$ when $\alpha^{\prime}=1$.

If $c=1$ we are done, so assume $c \geq 2$. Pick $\beta^{\prime}, \gamma \in K_{\ell}$ such that $\beta=$ $\beta^{\prime} \gamma^{p}$ and either $\beta^{\prime}=1$ or $\beta^{\prime}$ is $p$-primitive for $K_{\ell}$. Then $L^{\prime \prime}$ can be embedded into the compositum of the Galois closures $M$ of $K_{\max (\ell, c-1)}\left(\sqrt[p^{c-1}]{\beta^{\prime}}\right)$ and $M^{\prime}$ of $K_{\max (\ell, c-2)}\left(\sqrt[p^{c-2}]{\gamma}\right)$ over $K_{0}$. If $\beta^{\prime}=1$, then $h_{M / K_{0}}=\max (\ell-1, c-2) \leq \mu$. If $\beta^{\prime}$ is $p$-primitive for $K_{\ell}$, then $\beta^{\prime}=1+t_{\beta^{\prime}}$ with $v_{\ell}\left(t_{\beta^{\prime}}\right) \geq v_{\ell}\left(t_{\beta}\right)$. We then have, by Proposition 4.2, that $h_{M / K_{0}}=\max \left(\ell-1, c+\ell-2-\frac{v_{\ell}\left(t_{\beta^{\prime}}\right)-1}{p^{\ell-1}(p-1)}\right) \leq \mu$.

If $c=2$ we are done, so assume $c \geq 3$. By Lemma 1.1, it remains to prove that $h_{M^{\prime} / K_{0}} \leq \mu$. We prove by induction on $c$ that

$$
h_{M^{\prime} / K_{0}} \leq \max \left(\ell-1, c+\ell-3+\frac{1}{p^{\ell-1}(p-1)}\right),
$$

which is less than $\mu$ because $v_{\ell}\left(t_{\alpha^{\prime}}\right)<p^{\ell}$. Write $\gamma=\gamma^{\prime} \delta^{p}$, where either $\gamma^{\prime}=1$ or $\gamma^{\prime}$ is $p$-primitive for $K_{\ell}$. If $c=3$, then $M^{\prime}$ is the Galois closure of $K_{\max (\ell, c-2)}\left(\sqrt[p^{c-2}]{\gamma^{\prime}}\right)$ over $K_{0}$, and we conclude by Proposition 4.2. If $c>3$, then $M^{\prime}$ is contained in the compositum of the Galois closures $N$ of $K_{\max (\ell, c-2)}\left(\sqrt[p-2]{\gamma^{\prime}}\right)$ and $N^{\prime}$ of $K_{\max (\ell, c-3)}\left(\sqrt[p^{c-3}]{\delta}\right)$ over $K_{0}$. By Proposition 4.2. $h_{N / K_{0}} \leq \max (\ell-1, c+\ell-$ $\left.3+\frac{1}{p^{\ell-1}(p-1)}\right)$, and by the induction hypothesis, the same holds for $h_{N^{\prime} / K_{0}}$. We conclude using Lemma 1.1

The following version of Corollary 4.3 will be useful in $\$ 5$ and Obu09].

Corollary 4.4. Choose integers $\ell \geq 1$ and $c \geq 1$. Let $\alpha \in K_{\ell}$, not necessarily $p$ primitive for $K_{\ell}$, with $v_{\ell}(\alpha) \geq 0$. Write $L$ for the Galois closure of $K:=K_{d}\left(\sqrt[p^{c}]{\alpha}\right)$ over $K_{0}$, where $d \geq \max (\ell, c)$. Write $\alpha=1+t_{\alpha}$. Then

$$
h_{L / K_{0}} \leq \mu:=\max \left(d-1, c+\ell-1-\frac{v_{\ell}\left(t_{\alpha}\right)-1}{p^{\ell-1}(p-1)}, c+\ell-2+\frac{1}{p^{\ell-1}(p-1)}\right) .
$$

Proof. By Lemma 3.4 we have $v_{\ell}\left(t_{\alpha^{\prime}}\right) \geq v_{\ell}\left(t_{\alpha}\right)$, where $t_{\alpha^{\prime}}$ is from Corollary 4.3 . Furthermore, $v_{\ell}\left(t_{\beta}\right) \geq 0$, where $t_{\beta}$ is from Corollary 4.3. So our corollary follows from Corollary 4.3, along with Lemma 1.1 and the fact that $h_{K_{d} / K_{0}}=d-1$ (Proposition 1.4). 


\section{EXTENSIONS OF THE FORM $K_{c}\left(\sqrt[2^{c}]{a}\right)$}

In this section, we assume $p=2$. In order to understand the higher ramification groups above 2 in an extension $\mathbb{Q}\left(\zeta_{2^{c}}, \sqrt[2^{c}]{a}\right) / \mathbb{Q}$, when $a \in \mathbb{Q}$, it suffices, as mentioned in the introduction, to make a base change to the completion of the maximal unramified extension of $\mathbb{Q}_{2}$ (this is $K_{0}$, when $k=\overline{\mathbb{F}_{2}}$ ). We work in the more general context of an extension $K=K_{c}\left(\sqrt[2^{c}]{a}\right) / K_{0}$, with $a \in K_{0}$. Note that, since $p=2$, we have $K_{0}=K_{1}$, so the results of $\&$ apply.

By Proposition 1.3, in order to determine the higher ramification filtration of the Galois extension $K / K_{1}$, we need only determine the conductor of each Galois subextension $L$ of $K / K_{1}$. Each such subextension can be written in the form $L=K_{c^{\prime}}\left(\sqrt[2^{c^{\prime \prime}}]{a^{\prime}}\right)$, where $c^{\prime} \geq c^{\prime \prime}$ and $a^{\prime} \in K_{1}$. Then Lemma 1.1 implies that $h_{L / K_{1}}=\max \left(c^{\prime}-1, h_{L^{\prime} / K_{1}}\right)$, where $L^{\prime}=K_{c^{\prime \prime}}\left(\sqrt[2^{c^{\prime \prime}}]{a^{\prime}}\right)$. Since $L^{\prime} / K_{1}$ is in the same form as our original extension $K / K_{1}$, we content ourselves with finding the conductor $h_{K / K_{1}}$. For more details on the structure of subextensions of $K / K_{1}$, see e.g. [JV90, and dOV82.

After multiplying $a$ by an element of $\left(K_{1}^{\times}\right)^{2^{c}}$, which does not change the extension, we may assume that $0 \leq v_{K_{1}}(a)<2^{c}$. Write $a=2^{n} b$, where $v_{K_{1}}(b)=0$ and $0 \leq n<2^{c}$. After multiplying again by an element of $\left(K_{1}^{\times}\right)^{2^{c}}$, we may assume that $b \equiv 1(\bmod 2)$.

Theorem 5.1. Let $K=K_{c}\left(\sqrt[2^{c}]{a}\right)$, where $a=2^{n} b \in K_{0}=K_{1}$. Assume that $0 \leq n<2^{c}$ and $b \equiv 1(\bmod 2)$.

(i) If $n$ is odd, then $h_{K / K_{1}}=c+1$.

(ii) Suppose $2 \mid n$.

(a) If $c=1$ and $b \equiv 1(\bmod 4)$, then $K=K_{1}$.

(b) If $c=1$ and $b \equiv 3(\bmod 4)$, then $h_{K / K_{1}}=1$.

(c) If $4 \mid n, c>1$, and $b \equiv 1(\bmod 4)$, then $h_{K / K_{1}}=c-1$.

(d) If $4 \mid n, c>1$, and $b \equiv 3(\bmod 4)$, then $h_{K / K_{1}}=c$.

(e) If $4 \nmid n, c>1$, and $b \equiv 1(\bmod 4)$, then $h_{K / K_{1}}=c$.

(f) If $4 \nmid n, c=2$, and $b \equiv 3(\bmod 4)$, then $h_{K / K_{1}}=1$.

(g) If $4 \nmid n, c>2$, and $b \equiv 3(\bmod 4)$, then $h_{K / K_{1}}=c-\frac{1}{2}$.

Proof of (i). In this case, $a$ is 2-primitive for $K_{1}$, so Proposition 4.2 shows that $h_{K / K_{1}}=c+1$.

Proof of (ii)(a). By Epp73, §0.3], $2^{n} b$ is a square in $K_{1}$.

Proof of (ii)(b). This follows from Lemma 3.3(ii).

Proof of $(i i)(c)$. In this case, $K$ is contained in the compositum of $L:=K_{c}\left(\sqrt[2^{c}]{b}\right)$ and $L^{\prime}:=K_{c-2}\left(\sqrt[2^{c-2}]{2^{n / 4}}\right)$. Now, since $b \equiv 1(\bmod 4)$, it follows from [Epp73, §0.3] that $b$ is a square in $K_{1}$. So $L=K_{c}\left(\sqrt[2^{c-1}]{b^{\prime}}\right)$, where $b^{\prime 2}=b$ and $b^{\prime} \in K_{1}$. Since $b^{\prime} \equiv 1$ $(\bmod 2)$, it follows from Corollary 4.4 that $h_{L / K} \leq \max (c-1, c-1, c-1)=c-1$.

Also, by Corollary 4.3, $h_{L^{\prime} / K_{1}} \leq \max (\max (0, c-3), c-1, c-2)=c-1$. So $h_{K / K_{1}} \leq h_{L L^{\prime} / K_{1}} \leq c-1$, using Lemma 1.1. But $K$ contains $K_{c}$, and by Proposition 1.4 $h_{K_{c} / K_{1}}=c-1$. So $h_{K / K_{1}}=c-1$.

Proof of (ii) (d). Consider $L$ and $L^{\prime}$ as in (ii)(c). Since $b$ is 2-primitive for $K_{1}$, Proposition 4.2 gives us that $h_{L / K_{1}}=c$. We have seen in (ii)(c) that $h_{L^{\prime} / K_{1}} \leq c-1$. We conclude using Lemma 1.2 applied to the subextensions $L, L^{\prime}$, and $K$ of $L L^{\prime}$. 
Proof of $(i i)(e)$. In this case, $K$ is contained in the compositum of $L:=K_{c}\left(\sqrt[2^{c}]{b}\right)$ and $L^{\prime}:=K_{c-1}\left(\sqrt[2^{c-1}]{2^{n / 2}}\right)$. As in (ii)(c), $h_{L / K_{1}}=c-1$. Also, $2^{n / 2}$ is 2-primitive for $K_{1}$, so Proposition 4.2 (ii) shows that $h_{L^{\prime} / K_{1}}=c$. We conclude by applying Lemma 1.2 to the subextensions $L^{\prime}, L$, and $K$ of $L L^{\prime}$.

Proof of $(i i)(f)$. Since -4 is a 4 th power in $K_{2}$, we have that $K \cong K_{2}(\sqrt[4]{-b})$. Since $-b \cong 1(\bmod 4)$, the result follows from $($ ii $)(\mathrm{c})$.

Proof of $(i i)(g)$. Since $-4=(1+i)^{4}$ (where $i^{2}=-1$ ), it follows that $K$ is contained in the compositum of $L:=K_{c}\left(\sqrt[2^{c}]{-2^{n-2} b}\right)$ and the Galois closure $L^{\prime}$ of $K_{\max (2, c-2)}(\sqrt[2-2]{1+i})$ over $K_{1}$. By (ii) (c), $h_{L / K_{1}}=c-1$. Also, since $1+i$ is 2-primitive for $K_{2}$, Proposition 4.2 (ii) shows that $h_{L^{\prime} / K_{1}}=c-\frac{1}{2}$. We conclude using Lemma 1.2 applied to the subextensions $L^{\prime}, L$, and $K$ of $L L^{\prime}$.

\section{ACKNOWLEDGEMENTS}

The author thanks the referee for useful comments to improve the exposition. This work began as an appendix to Obu09]. The author also thanks the anonymous referee of Obu09] for suggesting that it be turned into its own paper.

\section{REFERENCES}

[dOV82] Mariá Acosta de Orozco and William Yslas Vélez, The lattice of subfields of a radical extension, J. Number Theory 15 (1982), no. 3, 388-405, DOI 10.1016/0022314X(82)90040-3. MR680540 (84e:12027)

[Epp73] Helmut P. Epp, Eliminating wild ramification, Invent. Math. 19 (1973), 235-249. MR.0321929 (48 \#294)

[JV90] Eliot T. Jacobson and William Y. Vélez, The Galois group of a radical extension of the rationals, Manuscripta Math. 67 (1990), no. 3, 271-284, DOI 10.1007/BF02568433. MR 1046989 (91j:11096)

[Obu09] Andrew Obus, Fields of moduli of three-point G-covers with cyclic p-Sylow. I, Algebra Number Theory 6 (2012), no. 5, 833-883, DOI 10.2140/ant.2012.6.833. MR2968628

[Obu10] Obus, Andrew. Fields of moduli of three-point G-covers with cyclic $p$-Sylow, II. To appear in J. Théor. Numbres Bordeaux.

[Ray99] Michel Raynaud, Spécialisation des revêtements en caractéristique $p>0$ (French, with English and French summaries), Ann. Sci. École Norm. Sup. (4) 32 (1999), no. 1, 87-126, DOI 10.1016/S0012-9593(99)80010-X. MR1670532 (2000e:14016)

[Ser79] Jean-Pierre Serre, Local fields, Graduate Texts in Mathematics, vol. 67, Springer-Verlag, New York, 1979. Translated from the French by Marvin Jay Greenberg. MR.554237 (82e:12016)

[Viv04] Filippo Viviani, Ramification groups and Artin conductors of radical extensions of $\mathbb{Q}$ (English, with English and French summaries), J. Théor. Nombres Bordeaux 16 (2004), no. 3, 779-816. MR2144967 (2006j:11148)

[Wew03b] Stefan Wewers, Three point covers with bad reduction, J. Amer. Math. Soc. 16 (2003), no. 4, 991-1032 (electronic), DOI 10.1090/S0894-0347-03-00435-1. MR.1992833 (2005f:14065)

Department of Mathematics, MC4403, Columbia University, 2990 Broadway, New York, New York 10027

Current address: Department of Mathematics, University of Virginia, P. O. Box 400137, Charlottesville, Virginia 229904-4137

E-mail address: obus@virginia.edu 